\title{
Errores de medicación en pacientes pediátricos en un hospital universitario en Medellín Colombia, un estudio de corte transversal
}

\author{
Andrés Felipe Valencia Quintero* \\ Juan Pablo Botero Aguirre** \\ Lina María González Santamaria*** \\ Pedro Amariles Muñoz ${ }^{* * * *}$ \\ Natalia Andrea Rojas Henao*****
}

\begin{abstract}
*Químico farmacéutico. Hospital Pablo Tobón Uribe. Integrante del Grupo de investigación Promoción y Prevención farmacéutica, Universidad de Antioquia. Medellín. Antioquia. Colombia.

**Químico farmacéutico. MSc en Epidemiología. Hospital Pablo Tobón Uribe. Medellín. Antioquia. Colombia.

***Enfermera. Hospital Pablo Tobón Uribe. Medellín. Antioquia. Colombia.

****Químico farmacéutico. PhD en Farmacia. Profesor Titular del Departamento de Farmacia, Universidad de Antioquia. Integrante Grupo de investigación Promoción y Prevención farmacéutica Universidad de Antioquia. Medellín. Antioquia. Colombia.

*****Química farmacéutica. MSc en Epidemiología. Hospital Pablo Tobón Uribe. Profesor cátedra. Departamento de Farmacia, Universidad de Antioquia. Medellín. Antioquia. Colombia.

Correspondencia: Sr. Valencia. Departamento de Servicios farmacéuticos, Hospital Pablo Tobón Uribe, Calle 78B \#69-240, Antioquia Medellín, Colombia. e-mail:valquife@gmail.com
\end{abstract}

Resumen

Introducción: Una tercera parte de los Errores en la atención en salud se ha relacionado a Errores de medicación; con una incidencia 3 veces mayor en pediatría con respecto a los pacientes adultos. Una estrategia recomendada para mejorar la gestión describe adoptar un sistema de notificación que permita la detección, identificación de causas e implementación de acciones enfocadas a la prevención. Objetivo: Determinar la prevalencia y caracterizar los errores de medicación en el servicio de pediatría reportados en el Sistema de notificación y gestión de riesgo clínico de un hospital universitario entre el 2017 y 2018. Metodología: Estudio de corte transversal. Se cuantificó la proporción de errores de medicación en el servicio de pediatría a partir de un informe del Sistema de notificación de riesgo clínico entre el año 2017 y 2018. Los errores de medicación se clasificaron por proceso de ocurrencia, tipo de error, subgrupo y gravedad. Para el análisis se utilizó estadística descriptiva. Resultados: Se generaron 669 reportes de eventos adversos, 376 (56,20\%) estaban relacionados con medicamentos. La tasa calculada de errores fue 7,71 por cada 1000 paciente-días. La mayoría de los errores de medicación se clasificaron como Error sin daño (categoría B y C), 176 ambas subcategorías (352 total) para un 93,62\% del total de errores. El proceso de prescripción reportó la mayoría de los errores 59,84\%. Conclusiones: La mayoría de los errores de medicación reportados se relacionaron con la prescripción, clasificándose principalmente como errores sin daño. MÉD.UIS.2020;33(2):33-40.

Palabras clave: Errores de medicación. Pediatría. Seguridad del paciente. Errores médicos. Gestión de riesgos.

\section{Medication errors in pediatric patients in an academic hospital in Medellin Colombia. A cross-sectional study}

Abstract

Introduction: A third of medical errors has been related to medication errors. In the pediatric population, an incidence of medication errors 3 times higher compared to adults has been described. A recommended strategy to improve medication errors risk management describes the adoption of a notification system that allows detection, identification of causes and the implementation of activities focused on prevention. Objective: To determine the prevalence and to characterize medication errors in the pediatric service reported in the clinical risk management and notification system of an academic hospital in 2017 and 2018. Methodology: Cross-sectional study. The proportion of medication errors in the pediatric department was quantified from a report of the Clinical Risk notification System for 2017 and 2018. Medication errors were grouped according to process, type of error, subgroup and gravity. Descriptive statistics were used for

Artículo recibido el 18 de noviembre de 2019 y aceptado para publicación el 29 de marzo de 2020. 
the analysis. Outcomes: 669 reports of adverse events were generated in the pediatric service, $376(56,20 \%)$ were related to medications. The error rate was 7,71 per-1000 patient days. Most of the errors were classified as Error without damage (category B and C), 176 both subcategories (total 352), representing $93,62 \%$ of the total of errors. The prescription process was the one that most reported errors $59,84 \%$. Conclusions: Most of the reported medication errors were related to the prescription. The main were classified in the errors without damage category. MÉD.UIS.2020;33(2):33-40.

Keywords: Medication errors. Pediatrics. Patient safety. Medical errors. Risk Management.

¿Cómo citar este artículo?: Valencia Quintero AF, Botero Aguirre JP, González Santamaria LM, Amariles Muñoz P, Rojas Henao NA. Errores de medicación en pacientes pediátricos en un hospital universitario en Medellín Colombia. Un estudio de corte transversal. MÉD.UIS.2020;33(2):33-40. doi: 10.18273/revmed.v33n2-2020004

\section{Introducción}

Los servicios de atención en salud tienen el objetivo de generar un beneficio para las personas hasta lograr su recuperación física, mental y social. Entre las actividades para cumplir este propósito es importante considerar la posibilidad de error en la práctica médica como un acto inherente a la condición humana que genera riesgo para la seguridad de los pacientes así lo concluyó un informe generado por el Instituto de Medicina de Los Estados Unidos (EE. UU.) a principios del año 2000 , este informe generó una alerta mundial al exponer que aproximadamente el $4 \%$ de los pacientes hospitalizados estarían en riesgo de sufrir algún daño por causas asociadas a la atención en salud, el 70\% generando incapacidad temporal y $14 \%$ eventos mortales causando entre 44000 y 98000 muertes, cifra situada por encima de la mortalidad producida por accidentes de tránsito, por cáncer de mama o por el SIDA'.

Un estudio un poco más reciente sitúo los Errores en la Atención en Salud (EAS) como la tercera causa de muerte en los EE. UU. después de enfermedades del corazón y cáncer ${ }^{2}$. Se ha descrito que cerca de la tercera parte de los EAS están relacionados a errores de medicación (EM) con una incidencia 3 veces mayor en la población pediátrica comparada con los adultos ${ }^{3,4}$. En Colombia la información sobre EM es limitada, algunos estudios describen una proporción de errores del $18,18 \%-45,69 \%^{11,20}$, sin embargo, no se conocen datos relacionados a la población pediátrica.

Los EM se pueden presentar en cualquiera de las etapas del proceso del uso de los medicamentos, desde la prescripción, administración, hasta el monitoreo del paciente5,6. Dentro de múltiples estrategias recomendadas por organismos internacionales para mejorar la seguridad del paciente, el Institute For Safe Medication Practices (ISMP),
American Hospital Association (AHA), el Institute for Healthcare Improvement (IHI) la Joint Commission International $(\mathrm{JCl})$, han recomendado la adopción de una cultura de seguridad enfocada a la mejora del sistema de atención, mediante la implementación de un programa voluntario, no punitivo, de notificación de EAS incluyendo EM, que permita la detección, análisis, identificación de causas e implementación de acciones enfocadas a su prevención?

El Hospital Pablo Tobón Uribe (HPTU) es un Hospital universitario de alta complejidad ubicado en la ciudad de Medellín-Colombia. Es una institución comprometida en velar por la seguridad en la atención de sus pacientes, acreditada por la $\mathrm{JCl}$, la cual tiene implementado un Sistema de notificación y gestión de riesgo clínico (SGRC) en el que se reportan de manera electrónica y voluntaria los eventos e incidentes relacionados con la atención en salud.

El objetivo de esta investigación fue determinar la prevalencia y caracterizar los errores de medicación reportados en el SGRC en el servicio de pediatría entre el 2017 y 2018.

\section{Materiales y métodos}

Estudio descriptivo de corte transversal. Se generó un informe de los EAS reportados en el sistema de gestión de riesgo clínico del HPTU durante los años 2017 y 2018. Para el análisis fueron incluidos los reportes asociados a medicamentos en el servicio de pediatría. Por otra parte, fueron excluidos aquellos reportes con datos incompletos. Los errores de medicación se clasificaron según la propuesta del grupo Ruiz-Jarabo $2000^{8}$ donde se catalogan por proceso de ocurrencia (prescripción, administración, conciliación, dispensación, monitorización y preparación), tipo de error, subgrupo y gravedad. 
Mayo - agosto

Los medicamentos involucrados en los reportes de EM fueron agrupados utilizando la clasificación anatómica, terapéutica y química (ATC, nivel 2) de la Organización Mundial de la Salud (OMS) y se identificaron los medicamentos de alto riesgo incluidos en el listado institucional siguiendo las recomendaciones del ISMP. Para controlar el sesgo de selección se incluyeron todos los reportes realizados en el servicio de pediatría, los investigadores tuvieron en cuenta que el personal que realiza el reporte recibió entrenamiento en el sistema de notificación como parte del plan de capacitación de la institución. La profesión o especialidad responsable de los reportes también fue registrada.

El registro y análisis de los datos se realizó en Stata ${ }^{\circledR}$ (StataCorp. 2013. Stata Statistical Software: Release 13. College Station, TX: StataCorp LP). Se emplearon estadísticos descriptivos para las variables cuantitativas y tablas de frecuencias para las variables cualitativas. Se calculó la proporción de EAS que corresponden a EM y la tasa de EM por cada 1000 paciente-días. El estudio fue aprobado por el comité de investigación y ética en investigación del HPTU.

\section{Resultados}

Se identificaron 13751 reportes de EAS en el sistema de gestión de riesgo clínico del HPTU; 4589 (33,37\%) correspondieron a eventos relacionados con medicamentos. En el servicio de hospitalización pediátrica se generaron 669 reportes de EAS, 376 $(56,20 \%)$ relacionados con medicamentos. La tasa
Errores de medicación en pacientes pediátricos en un hospital universitario en Medellín Colombia. Un estudio de corte transversal

calculada de EM fue 7,71 por cada 1000 paciente-días. La mayoría de los errores reportados se clasificaron como error sin daño (categorías B y C) con un total de 176 ambas subcategorías (352 total) para un $93,62 \%$ del total de errores. Se reportó 1 error con daño (categoría E) relacionado con un paciente con diagnóstico de tuberculosis que requirió cambio de tratamiento antituberculoso por un esquema alterno debido a monitorización insuficiente y falta de control de pruebas hepáticas.

El proceso de prescripción fue el que más reportó errores con un total de 225 (59,84\%), seguido de administración con 78 (20,74\%) (Ver Tabla 1). La dosis incorrecta fue el subgrupo de error más común tanto en el proceso de prescripción como de administración, $134(59,56 \%)$ y $13(16,67 \%)$, respectivamente. (Ver Tabla 2).

Los medicamentos de alto riesgo estuvieron involucrados en $62(16,49 \%)$ reportes. El grupo terapéutico con mayor número de reportes fue los antibacterianos de uso sistémico (J01) con 99 (26,32\%), seguido de analgésicos (No2) 44 (11,70\%) y antiepilépticos (No3) 31 (8,24\%). Los demás grupos terapéuticos reportados se presentan en la Figura 1. El $16.49 \%$ de los medicamentos eran de alto riesgo.

Los químicos farmacéuticos fueron los profesionales que más reportes generaron 196 (52,13\%), seguido en orden descendente por enfermería 123 (32,71\%), regencia de farmacia $38(10,11 \%)$, medicina $7(1,86 \%)$, auxiliar de enfermería 4(1,06\%). En 8 reportes $(2,13 \%)$ no se registró el nombre de la especialidad.

Tabla 1. Errores de medicación de acuerdo con el proceso en el que se presentaron y su gravedad.

\begin{tabular}{|c|c|c|c|c|c|c|c|c|}
\hline \multirow{2}{*}{\multicolumn{2}{|c|}{ *Categoría de gravedad }} & \multicolumn{6}{|c|}{ Proceso implicado } & \multirow{3}{*}{$\begin{array}{c}\begin{array}{c}\text { Total general } \\
\mathbf{n}(\%)\end{array} \\
19(5,05)\end{array}$} \\
\hline & & \multirow{2}{*}{$\begin{array}{c}\text { Prescripción } \\
12\end{array}$} & \multirow{2}{*}{$\begin{array}{c}\text { Administración } \\
4\end{array}$} & \multirow{2}{*}{\begin{tabular}{|c} 
Conciliación \\
-
\end{tabular}} & \multirow{2}{*}{$\begin{array}{c}\text { Dispensación } \\
-\end{array}$} & \multirow{2}{*}{$\begin{array}{c}\begin{array}{c}\text { Monitorización } \\
\text { del paciente o } \\
\text { del tratamiento }\end{array} \\
3\end{array}$} & \multirow{2}{*}{ Preparación } & \\
\hline $\begin{array}{l}\text { Error } \\
\text { potencial }\end{array}$ & Categoría A & & & & & & & \\
\hline \multirow{3}{*}{$\begin{array}{l}\text { Error sin } \\
\text { daño }\end{array}$} & Categoría B & 140 & 3 & 10 & 1 & 18 & 4 & $176(46,81)$ \\
\hline & Categoría C & 72 & 70 & 4 & 13 & 5 & 12 & $176(46,81)$ \\
\hline & Categoría D & 1 & 1 & -- & -- & -- & 2 & $4(1,06)$ \\
\hline \multirow[t]{2}{*}{$\begin{array}{l}\text { Error con } \\
\text { daño }\end{array}$} & Categoría E & -- & -- & - & - & 1 & - & $1(0,27)$ \\
\hline & $\begin{array}{l}\text { Total general } \\
\mathrm{n}(\%)\end{array}$ & $225(59,84)$ & $78(20,74)$ & $14(3,72)$ & $14(3,72)$ & $27(7,18)$ & $18(4,79)$ & $376(100)$ \\
\hline
\end{tabular}

*Clasificación adaptada y actualizada del National Coordinating Council for Medication Error

Fuente: autores 
Valencia Quintero AF, Botero Aguirre JP, González Santamaria LM, Amariles Muñoz P,

Rojas Henao NA.

MÉD.UIS. 2020;33(2):33-40

Tabla 2. Errores de medicación de acuerdo con el proceso y subgrupo de error.

\begin{tabular}{|c|c|c|}
\hline *Proceso/Tipo de error & *Subgrupo del error & Total $n(\%)$ \\
\hline \multicolumn{2}{|r|}{ Prescripción } & 225 \\
\hline \multirow{3}{*}{ Dosis Incorrecta } & & $134(59,56)$ \\
\hline & Dosis mayor & 83 \\
\hline & Dosis menor & 51 \\
\hline \multirow{2}{*}{ Duración del tratamiento incorrecta } & & $1(0,44)$ \\
\hline & Duración mayor & 1 \\
\hline \multicolumn{2}{|l|}{ Forma farmacéutica errónea } & $5(2,22)$ \\
\hline \multicolumn{2}{|l|}{ Frecuencia de administración errónea } & $16(7,11)$ \\
\hline \multirow{5}{*}{ Medicamento erróneo } & & $48(21,33)$ \\
\hline & Alergia previa o efecto adverso similar & 22 \\
\hline & Duplicidad terapéutica & 21 \\
\hline & $\begin{array}{l}\text { Medicamento inapropiado para el paciente por edad, situación clínica, } \\
\text { etc. }\end{array}$ & 3 \\
\hline & Medicamento no indicado/apropiado para el diagnóstico & 2 \\
\hline \multirow{2}{*}{ Omisión de dosis o de medicamento } & & $19(8,44)$ \\
\hline & Falta de prescripción de un medicamento necesario & 19 \\
\hline \multicolumn{2}{|l|}{ Prescripción inapropiada } & $1(0,44)$ \\
\hline \multicolumn{2}{|l|}{ Velocidad de administración errónea } & $1(0,44)$ \\
\hline & Administración & 78 \\
\hline \multirow{4}{*}{ Dosis Incorrecta } & & $13(16,67)$ \\
\hline & Dosis extra & 3 \\
\hline & Dosis mayor & 5 \\
\hline & Dosis menor & 5 \\
\hline \multirow{2}{*}{ Duración del tratamiento incorrecta } & & $2(2,56)$ \\
\hline & Duración mayor & 2 \\
\hline \multicolumn{2}{|c|}{ Error de preparación/manipulación/acondicionamiento } & $1(1,28)$ \\
\hline \multicolumn{2}{|l|}{ Forma farmacéutica errónea } & $2(2,56)$ \\
\hline \multicolumn{2}{|l|}{ Frecuencia de administración errónea } & $6(7,69)$ \\
\hline \multicolumn{2}{|l|}{ Hora de administración incorrecta } & $6(7,69)$ \\
\hline \multirow{4}{*}{ Medicamento erróneo } & & $12(15,38)$ \\
\hline & Interacción medicamento-medicamento & 1 \\
\hline & $\begin{array}{l}\text { Medicamento inapropiado para el paciente por edad, situación clínica, } \\
\text { etc. }\end{array}$ & 2 \\
\hline & $\begin{array}{l}\text { Transcripción/dispensación/administración de un medicamento } \\
\text { diferente al prescrito }\end{array}$ & 9 \\
\hline \multirow{3}{*}{ Omisión de dosis o de medicamento } & & $23(29,49)$ \\
\hline & Falta de prescripción de un medicamento necesario & 1 \\
\hline & Omisión en la administración & 22 \\
\hline \multicolumn{2}{|l|}{ Paciente equivocado } & $6(7,69)$ \\
\hline \multicolumn{2}{|l|}{ Técnica de administración incorrecta } & $1(1,28)$ \\
\hline \multicolumn{2}{|l|}{ Velocidad de administración errónea } & $3(3,85)$ \\
\hline \multicolumn{2}{|l|}{ Vía de administración errónea } & $3(3,85)$ \\
\hline \multicolumn{2}{|r|}{ Conciliación } & 14 \\
\hline \multirow{3}{*}{ Dosis Incorrecta } & & $11(78,57)$ \\
\hline & Dosis mayor & 3 \\
\hline & Dosis menor & 8 \\
\hline
\end{tabular}


Mayo - agosto

Errores de medicación en pacientes pediátricos en un hospital universitario en Medellín Colombia. Un estudio de corte transversal

\begin{tabular}{|c|c|c|}
\hline *Proceso/Tipo de error & *Subgrupo del error & Total $\mathrm{n}(\%)$ \\
\hline Frecuencia de administración errónea & & $2(14,29)$ \\
\hline \multicolumn{2}{|l|}{ Medicamento erróneo } & $1(7,14)$ \\
\hline & Dispensación & 14 \\
\hline \multirow[t]{2}{*}{ Dosis Incorrecta } & & $1(7,14)$ \\
\hline & Dosis menor & 1 \\
\hline $\begin{array}{l}\text { Error de preparación/manipulación/ } \\
\text { acondicionamiento }\end{array}$ & & $1(7,14)$ \\
\hline Frecuencia de administración errónea & & $1(7,14)$ \\
\hline \multirow[t]{2}{*}{ Medicamento erróneo } & & $10(71,43)$ \\
\hline & $\begin{array}{l}\text { Transcripción/dispensación/administración de un medicamento } \\
\text { diferente al prescrito }\end{array}$ & 10 \\
\hline \multirow{2}{*}{ Omisión de dosis o de medicamento } & & $1(7,14)$ \\
\hline & Omisión en la dispensación & 1 \\
\hline \multicolumn{2}{|c|}{ Monitorización del paciente o del tratamiento } & 27 \\
\hline \multirow{2}{*}{ Medicamento erróneo } & & $1(3,70)$ \\
\hline & Interacción medicamento-medicamento & 1 \\
\hline \multirow{2}{*}{ Monitorización insuficiente del tratamiento } & & $23(85,18)$ \\
\hline & Falta de controles analíticos & 23 \\
\hline \multirow{2}{*}{ Otros (texto libre) } & & $3(11,11)$ \\
\hline & Tiempo de muestra de laboratorio equivocada & 3 \\
\hline & Preparación & 18 \\
\hline \multirow{3}{*}{ Dosis Incorrecta } & & $2(11,11)$ \\
\hline & Dosis mayor & 1 \\
\hline & Dosis menor & 1 \\
\hline \multicolumn{2}{|c|}{ Error de preparación/manipulación/acondicionamiento } & $13(72,22)$ \\
\hline \multirow[b]{2}{*}{ Medicamento erróneo } & & $2(11,11)$ \\
\hline & $\begin{array}{l}\text { Transcripción/dispensación/administración de un medicamento } \\
\text { diferente al prescrito }\end{array}$ & 2 \\
\hline \multirow{2}{*}{ Omisión de dosis o de medicamento } & & $1(5,56)$ \\
\hline & Falta de prescripción de un medicamento necesario & 1 \\
\hline
\end{tabular}

*Clasificación adaptada y actualizada del National Coordinating Council for Medication Error Reporting and Prevention (NCC MERP)

Fuente: autores.

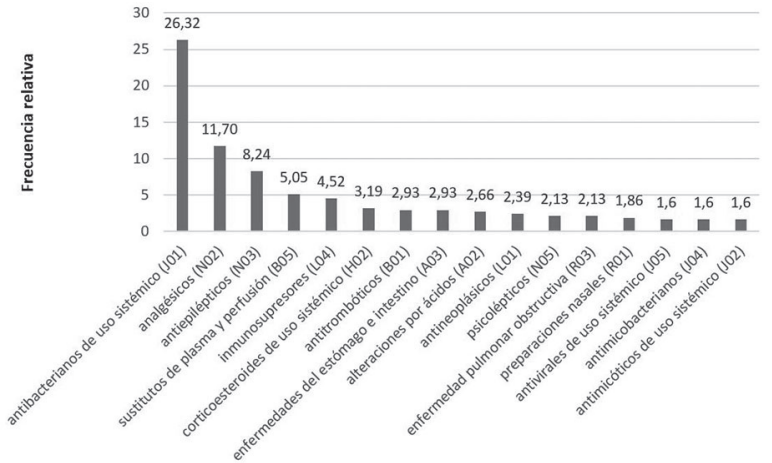

Figura 1. Distribución de los medicamentos involucrados en los EM reportados en el SGRC

Fuente: autores.

\section{Discusión}

La proporción global de errores de medicación varía en la literatura publicada, sin embargo, conocer su prevalencia, permite implementar acciones enfocadas a su prevención y control' 7 . En Colombia no se conocen datos de estudios que reporten errores de medicación en la población pediátrica, por ello, los resultados reportados en el presente estudio evidencian una problemática que prevalece a nivel global afectando los sistemas de salud y la calidad en la atención del paciente9.

La proporción de EM encontrados en el presente trabajo son consistentes con los datos reportados por el estudio ENEAS el cual fue realizado en España 
buscando conocer los efectos adversos ligados a la hospitalización reportando una proporción de $37,40 \%$ relacionados con medicamentos ${ }^{10}$. Un estudio que caracterizó los reportes de incidentes del programa de seguridad del paciente en un hospital de alta complejidad describió el $18.80 \%$ relacionados a $E M^{11}$. Datos del Reino Unido mostraron que 517415 notificaciones de incidentes de medicación en Inglaterra y Gales correspondieron a un 10\% del total de reportes de seguridad del paciente ${ }^{12}$. Los anteriores estudios mencionados describen datos de EM en general sin discriminar los presentados en la población pediátrica.

En la presente investigación la proporción de EM en el servicio de hospitalización de pediatría fue $56,20 \%$. Este resultado difiere con lo reportado por Shaw et al. y Otero y et al. quienes, en sus trabajos realizados sobre errores de medicación en niños, encontraron una proporción de EM de 19,22\% y $11,40 \%$ respectivamente ${ }^{13-14}$. La diferencia entre los datos reportados en los estudios se puede explicar por las diferentes metodologías empleadas para recolectar la información. Algunos, incluyendo esta investigación, describen en su metodología la revisión de notificación voluntaria de incidentes por el personal asistencial, otros la revisión y seguimiento de la historia clínica de los pacientes en busca de los errores ${ }^{12-14}$. Con relación a lo anterior, es preciso mencionar que estos métodos de identificación de EM son complementarios y ninguno de ellos tiene la capacidad de detectar la totalidad de los incidentes de medicación que se producen dada la complejidad del sistema de utilización del medicamento ${ }^{12}$.

La literatura reporta una incidencia de EM en la población pediátrica dos a tres veces mayor comparado con adultos ${ }^{15}$. Los datos del presente estudio se aproximan a esta cifra, reportando una proporción de EM de $32,20 \%$ en pacientes adultos versus $56,20 \%$ en pacientes pediátricos, siendo este valor 1,74 veces superior en los niños. El aumento en la probabilidad de cometerse un error en pediatría se ha relacionado con la falta de aprobación para el uso en esta población de gran parte de los medicamentos existentes en el mercado, lo que conlleva a extrapolarse la información descrita para pacientes adultos, adicional a la necesidad de la realización de cálculos para la dosificación de acuerdo con el peso, edad, e incluso superficie de área corporal en algunos casos. Por lo anterior, para mitigar este riesgo se requiere la constante capacitación, entrenamiento, evaluación y seguimiento al personal de salud involucrado en el proceso de atención del paciente pediátrico, la estandarización y realización de protocolos, así como aprender acerca de los EM reportados ${ }^{3,16}$.

La tasa de EM reportada en la literatura es variable, Peter y col en una revisión sistemática y metanálisis sobre errores de medicación en pacientes pediátricos hospitalizados reportó un valor de 62,80 errores de medicación por cada 1000 paciente-días ${ }^{17}$, en Argentina otro estudio reportó una tasa de 130,60 por cada 1000 paciente-días ${ }^{18}$, los datos reportados en el presente estudio son inferiores, con una tasa de 7,71 por cada 1000 paciente-días.

Los resultados encontrados en esta investigación sobre la gravedad de los EM son similares al estudio de Guerrero-Aznar MD et al., quienes señalan que los errores de medicación en pacientes pediátricos notificados a la comisión central de seguridad de Andalucía se clasificaron en la categoría $\mathrm{B}^{19}$.

En el HPTU el proceso con mayor porcentaje de EM reportados fue la prescripción, similar a lo encontrado por Otero et al. y Kaushal et al. que encontraron un proporción de errores de prescripción de 56 y 78\% respectivamente ${ }^{14,20}$.

Un estudio realizado en Estados Unidos evaluó el proceso de prescripción de medicamentos, encontrando al igual que los datos del HPTU la dosis incorrecta como el error de mayor proporción $58.96 \%{ }^{16}$, adicional este estudio sugiere que los errores se pueden presentar al realizar cálculos con relación al peso y/o superficie corporal de los pacientes, realizar conversión de unidades (mililitros a gotas, microgramos a gramos, miliequivalentes a miligramos) y ajustar la dosificación cuando la función renal o hepática del paciente se ha deteriorado ${ }^{21}$.

La administración fue el segundo proceso con mayor proporción de EM detectados relacionándose la mayoría con omisión de dosis diferente a lo encontrado en Malasia en un estudio que evaluó EM relacionados con la administración que reportó la mayoría (34,62\%) como aquellos en los que se administraron los medicamentos en una concentración inadecuada ${ }^{22}$.

Los medicamentos con mayor número de reportes fueron los antibacterianos de uso sistémico (26,32\%), similar a lo reportado por Guerrero Aznar y col. en un estudio de corte transversal donde encontraron 
Mayo - agosto

el $31,21 \%$ de los EM relacionados con este grupo terapéutico ${ }^{19}$. Dicho grupo farmacológico cobra importancia debido al uso racional que se les debe dar para disminuir el riesgo de resistencia bacteriana, en el HPTU la mayoría de los errores se relacionaron con el monitoreo de concentraciones séricas para establecer un régimen de dosificación efectivo y seguro.

El 52,13\% de los reportes generados por los químicos farmacéuticos contrasta con la literatura, un estudio en Estados unidos identificó a las enfermeras como la población clínica que más reportes de EM generaba con un $98,32 \%$, mientras que el reporte por otro tipo de profesionales sólo llegaba al $38,15 \% 23$, para Ross y col en el Reino Unido el 59,22\% de los reportes generados correspondió a enfermeras ${ }^{24}$, en nuestro estudio el 32,71\% de los reportes fueron generados por estos profesionales y el personal médico se situó dentro de los que menos generaron reportes (1,86\%), similar a lo mostrado por Farley et al. donde hallaron entre $1-2 \%$ de los EM reportados por médicos ${ }^{25}$.

El presente estudio tiene limitaciones. Los datos provienen de una sola institución lo cual limita la generalización de los resultados, además fueron obtenidos de un sistema electrónico de notificación voluntaria. Se ha descrito que este tipo de notificación presenta subregistro debido a que solo se reportan errores considerados importantes que generan daño o cambios en las conductas de la atención, quedando omitidos los errores que no generan daño, errores potenciales, errores identificados pero considerados insignificantes, así como los errores con potencial de generar daño ${ }^{25}$. Pese a lo anterior, los sistemas de notificación voluntaria son recomendados como estrategia de seguridad en la atención del paciente por su relativa facilidad para la implementación, además porque permiten identificar riesgos, causas, así como generar acciones enfocadas a la prevención favoreciendo un entorno seguro para el paciente?

\section{Conclusiones}

La mayoría de los EM reportados en el SGRC del hospital en el servicio de hospitalización de pediatría se relacionaron con la prescripción y en segundo lugar la administración, siendo los errores sin daño la principal categoría.

La prevalencia de EM en pediatría encontrada en el presente estudio fue mayor al compararse con los
Errores de medicación en pacientes pediátricos en un hospital universitario en Medellín Colombia. Un estudio de corte transversal

datos en pacientes adultos consecuentemente con lo reportado en la literatura.

Se recomienda la implementación de un sistema de notificación voluntaria de incidentes como estrategia para la identificación de riesgos en la atención en salud y Errores de medicación.

\section{Conflicto de intereses}

El trabajo fue realizado con recursos propios del Hospital Pablo Tobón Uribe. No requirió financiación externa. Los autores declaran no tener conflicto de intereses.

\section{Referencias bibliográficas}

1. Kohn L, Corrigan J, Donaldson M. To Err is Human: Building a Safer Health System. Washington, DC: National Acad Press; 2008.

2. Makary MA, Daniel M. Medical error-the third leading cause of death in the US. BMJ (Online). 2016;353:i2139.

3. Gutiérrez S, Mogni A, Berón A, Iramain R. Errores de medicación en niños hospitalizados. Arch Pediatría Urug. 2011;82(3):133-40.

4. Miller M, Robinson K, Lubomski L, Rinke M, Pronovost P. Medication errors in paediatric care: a systematic review of epidemiology and an evaluation of evidence supporting reduction strategy recommendations. Qual Saf Health Care. 2007;16(2):11626.

5. Bates DW, Cullen DJ, Laird N, Petersen LA, Small SD, Servi D, et al. Incidence of adverse drug events and potential adverse drug events. Implications for prevention. ADE Prevention Study Group. JAMA. 1995;274(1):29-34.

6. Sánchez-Muñoz LA, Mayor-Toranzo E, Rodríguez-Martín C. Análisis modal de fallos y efectos del sistema de utilización de medicamentos. Farm Hosp. 2012;36(4):299-300.

7. Otero López MJ. Nuevas iniciativas para mejorar la seguridad de la utilización de los medicamentos en los hospitales. Rev Esp Salud Pública. 2004;78(3):323-39.

8. Otero López MJ, Castaño Rodríguez B, Pérez Encinas M, Codina Jané C, TamésAlonso MJ, Sánchez Muñoz T. Actualización de la clasificación de errores de medicación del grupo Ruiz-Jarabo 2000. Farm Hosp. 2008;32(1):38-52.

9. World Health Organization. WHO launches global effort to halve medication-related errors in 5 years [Internet]. 2017 [citado 28 abril 2019]. Disponible en: https://www.who.int/newsroom/detail/29-03-2017-who-launches-global-effort-to-halvemedication-related-errors-in-5-years

10. Pastó-Cardona L, Masuet-Aumatell C, Bara-Oliván B, CastroCels I, Clopés-Estela A, Pàez-Vives F, et al. Estudio de incidencia de los errores de medicación en los procesos de utilización del medicamento: prescripción, transcripción, validación, preparación, dispensación y administración en el ámbito hospitalario. Farm Hosp. 2009;33(5):257-68.

11. Cortázar Y. Análisis de los reportes de errores de medicación de un programa de seguridad de paciente en un hospital de alta complejidad de la ciudad de Bogotá [tesis de maestría]. Universidad Nacional de Colombia; 2018. Recuperado de https://repositorio.unal.edu.co/bitstream/handle/unal/69212/ tesisyira\%20ver\%20final.pdf?sequence $=1$ \&isAllowed $=\mathrm{y}$

12. Organización Mundial de la Salud. Sistemas de notificación y aprendizaje sobre errores de medicación: el papel de los centros de farmacovigilancia[Internet]. . Ginebra: Organización Mundial de la Salud; 2018. Disponible en: https://apps.who.int/iris/ bitstream/handle/10665/276898/9789243507941-spa.pdf?ua=1

13. Shaw, K. N. et al. Reported medication events in a paediatric 
emergency research network: Sharing to improve patient safety. Emergency Medicine Journal. 2013; 30 (10): 815-9.

14. Otero, P., Leyton, A., Mariani, G. \& Cernadas, J. M. C. Medication errors in pediatric inpatients: Prevalence and results of a prevention program. Pediatrics. 2008; 122(3): 737-43.

15. Assunção Costa L, Valli C, Pimentel Alvarenga A. Erros de dispensação de medicamentos em um hospital público pediátrico. Rev Latino-am. Enfermagem. 2008;16(5).

16. Honey BL, Bray WM, Gomez MR, Condren M. Frequency of prescribing errors by medical residents in various training programs. J Patient Saf. 2015;11(2):100-4.

17. Gates, P. J. et al. Prevalence of Medication Errors Among Paediatric Inpatients: Systematic Review and Meta-Analysis. Drug Safety. 2019; 42 (11) 1329-1342.

18. Ocaña V, Sánchez Domenech G, Sánchez C. Errores en el proceso de medicación de pacientes internados en el Hospital Público Materno Infantil de Salta. Rev Argent Salud Pública. 2017; 8(31): 19-26.

19. Guerrero-Aznar MD, Jiménez-Mesa E, Cotrina-Luque J, Villalba-Moreno A, Cumplido-Corbacho R, FernándezFernández L. Validation of a methodfornotifying and monitoringmedicationerrors in pediatrics. AnPediatr (Barc).
2014; 81:360-367.

20. Kaushal R. Medication Errors and Adverse Drug Events in Pediatric Inpatients. JAMA. 2001;285(16):2114.

21. Hoyle JD, Davis AT, Putman KK, Trytko JA, Fales WD. Medication dosing errors in pediatric patients treated by emergency medical services. Prehospital Emerg Care Off J Natl Assoc EMS Physicians Natl Assoc State EMS Dir. 2012;16(1):59-66.

22. Chua SS, Chua HM, Omar A. Drug administration errors in paediatric wards: a direct observation approach. Eur J Pediatr. 2010;169(5):603-11.

23. Farley DO, Haviland A, Champagne S, Jain AK, Battles JB, Munier WB, et al. Adverse-event-reporting practices by US hospitals: results of a national survey. Qual Saf Health Care. 2008;17(6):416 23.

24. Ross LM, Wallace J, Paton JY. Medication errors in a paediatric teaching hospital in the UK: five years operational experience. Arch Dis Child. 2000;83(6):492-7.

25. Chi Man C. Public Opinions Help Those Who Help Themselves: ¿Do Doctors Deserve the Right to Dispense? [Internet]. The Hong Kong Medical Association; 2006 [citado 25 de julio de 2019]. Disponible en: http://www.hkma.org/english/cme/onlinecme/ cme200610set.htm 